\title{
A VARIATIONAL METHOD FOR THE LIMIT ANALYSIS OF ANISOTROPIC PLATES*
}

BY

\author{
S. L. LEE, T. MURA Northwestern University \\ AND
}

J. S. KAO Marquette University

1. Introduction. It has been shown by Mura and Lee [1] that the safety factor, the kinematically admissible multiplier and the statically admissible multiplier in the limit analysis of perfectly plastic solids are actually the extremum values of the same functional under different constraint conditions. Due to the complexity of these constraint conditions, however, the application of this functional to the calculation of the upper and lower bounds requires further simplification in the mathematical procedure. In this paper, some of the constraint conditions are removed and the upper and lower bounds are calculated respectively by means of two new functionals which have been extremized by variational procedure. The application of these functionals are illustrated by means of a simply supported circular orthotropic plate subjected to uniformly distributed load. The results obtained by the proposed method are compared with those obtained by the classical method.

2. Safety factor. The safety factor of an anisotropic plate for a given load $p$ is the stationary value of the following functional [1]

$$
\begin{aligned}
F\left[\dot{w}, M_{i i}, R, m, \mu, \phi\right]= & \int_{A} M_{i i}\left(-\dot{w}_{, i}\right) d A-\int_{S} R \dot{w} d S \\
& -m\left(\int_{A} p \dot{w} d A-1\right)-\int_{A} \mu\left[f\left(M_{i j}\right)+\phi^{2}\right] d A
\end{aligned}
$$

with the constraint condition

$$
\mu \geq 0
$$

where

$$
f\left(M_{i j}\right)=\frac{1}{2} C_{i j k l} M_{i j} M_{k l}-k^{2}
$$

and, $j$ denotes differentiation with respect to $x_{i}$. In these equations, $\dot{w}_{, i}$ denotes the curvature rate field associated with the velocity field $\dot{w}, M_{i i}$ the bending moment field, $k$ the yield parameter, $A$ the domain, and $S$ the boundary of the domain. Also $C_{i i k l}$ are the material constants, $\phi$ is a point function and $R, m$ and $\mu$ are Lagrangian multipliers.

The proof of the above statement is given as follows. Taking the variation of $F$ leads to the following natural conditions:

*Received February 17, 1966; revised manuscript received June 13, 1966. The research upon which this paper is based was supported in part by the National Science Foundation under Grant No. GP 2094 to Northwestern University. 


$$
\begin{aligned}
& -\dot{w}_{, i j}=\mu \partial f / \partial M_{i j}, \quad \mu \geq 0, \quad \text { in } A, \\
& M_{i, i i}=-m p \quad \text { in } A \text {, } \\
& M_{i j} n_{i} \delta \dot{w}_{, i}=0 \quad \text { on } S \text {, } \\
& M_{i, j} n_{i}=R \quad \text { on } S \text {, } \\
& f\left(M_{i i}\right)+\phi^{2}=0 \quad \text { in } A \text {, } \\
& \mu \phi=0 \quad \text { in } A \text {, } \\
& \dot{w}=0 \quad \text { on } S, \\
& \int_{A} p \dot{w} d A=1 .
\end{aligned}
$$

Condition (4) is the plastic potential flow law, (5) to (7) are the equilibrium and boundary conditions, (8) and (9) define the admissible domain of the stress fields, i.e.,

$$
\begin{aligned}
& f\left(M_{i j}\right)=0, \quad \text { if } \quad \mu>0, \\
& f\left(M_{i j}\right) \leq 0, \quad \text { if } \quad \mu=0,
\end{aligned}
$$

and conditions (10) and (11) define the velocity field. It should be observed that the left side of (6) can be written in the form

$$
M_{n} \partial(\delta \dot{w}) / \partial n+M_{n s} \partial(\delta \dot{w}) / \partial s=0, \quad \text { on } S
$$

where $M_{n}$ and $M_{n s}$ are the bending and twisting moments at the boundary respectively. Since the slope tangential to the boundary is zero from (10), (13) becomes

$$
M_{n} \partial(\delta \dot{w}) / \partial n=0, \quad \text { on } S .
$$

When the plate is simply supported, (14) leads to

$$
M_{n}=0, \quad \text { on } S
$$

since $\partial(\dot{w}) / \partial n$ is arbitrary in this case. If the plate is clamped, (14) becomes

$$
\partial(\dot{w}) / \partial n=0, \quad \text { on } S .
$$

It is obvious that conditions (4) to (11) are the conditions for incipient plastic flow. Integrating the functional by parts in view of (4) to (11), it can be readily shown that the extremum value of $F$ is equal to $m$. Therefore the safety factor $s$ can be defined as the extremum value of $F$, i.e.,

$$
\text { Ext } F=m=s
$$

3. Lower bound theorem. A lower bound of the safety factor is

$$
m^{0}=m / \alpha^{0}
$$

where

$$
\alpha^{0}=\left[\frac{k^{2}}{\max \left\{\frac{1}{2} C_{i j k l} M_{i j} M_{k l}\right\}}\right]^{1 / 2} .
$$

In the above equations, $m$ and $M_{i j}$ are obtained by the direct method of variational 
calculus using the following functional with arguments $m, M_{i i}, \mu$ and $\phi$ :

$$
F^{0}=m-\int_{A} \mu\left[f\left(M_{i j}\right)+\phi^{2}\right] d A
$$

with constraint conditions (2) and (5) for clamped boundary, and conditions (2), (5) and (15) for simply supported boundary. The above functional is derived by integrating (1) by parts in view of (5), (6) and (7). The stress field $M_{i j} / \alpha^{0}$ is statically admissible since

$$
\begin{gathered}
\left(M_{i i} / \alpha^{0}\right)_{, i i}=-\left(m / \alpha^{0}\right) p, \\
\frac{1}{2} C_{i j k l}\left(M_{i j} / \alpha^{0}\right)\left(M_{k l} / \alpha^{0}\right) \leq k^{2}, \\
M_{n} / \alpha^{0}=0, \quad \text { on } S
\end{gathered}
$$

the last condition applicable only to simply supported boundary.

4. Upper bound theorem. An upper bound of the safety factor is

$$
m^{*}=2 k^{2} \int_{A} \mu \alpha^{*} d A
$$

where $\alpha^{*}$ is a point function defined by

$$
\frac{1}{2} C_{i j k l} M_{i j} M_{k l}=\left(\alpha^{*}\right)^{2} k^{2} .
$$

In the above equations, $\mu$ and $M_{i i}$ are obtained by the direct method of variational calculus using the following functional with arguments $\mu, M_{i}$ and $\phi$ :

$$
F^{*}=2 k^{2} \int_{A} \mu d A+\int_{A} \mu\left[f\left(M_{i j}\right)-\phi^{2}\right] d A
$$

with constraint conditions (2), (4), (10) and (11) for simply supported boundary, with the addition of condition (16) for clamped boundary. The above functional is derived from (1) in view of these constraint conditions and the fact that $M_{i j} \partial f / \partial M_{i j}=2 f+2 k^{2}$.

In order to prove that (24) is an upper bound, consider the following expression for the safety factor:

$$
s=\int_{A} \bar{M}_{i j}\left(-\dot{w}_{, i j}\right) d A
$$

where $\bar{M}_{i i}$ is the true stress field and $\dot{w}$ is a velocity field associated with (26) and the constraint conditions. In view of condition (4), the right-hand side in (27) becomes

$$
\int_{A} \mu C_{i j k l} M_{k l} \bar{M}_{i j} d A
$$

an application of Schwartz's inequality in Riemann space with fundamental tensors $C_{i j k l}$ leads to

$$
s \leq \int_{A} \mu\left(C_{i j k l} M_{i j} M_{k l}\right)^{1 / 2}\left(C_{i j k l} \bar{M}_{i j} \bar{M}_{k l}\right)^{1 / 2} d A .
$$

In view of (25) and the fact that $\frac{1}{2} C_{i j k l} \bar{M}_{i i} \bar{M}_{k l} \leq k^{2}$, (20) becomes

$$
s \leq 2 k^{2} \int_{A} \mu \alpha^{*} d A \text {. }
$$


5. Example. Consider a simply supported circular orthotropic plate of radius $a$, subjected to a uniformly distributed load of intensity $p$, and assume that the principal directions of orthotropy are parallel to the axes $\theta=0$ and $\theta=\pi / 2$ (Fig. 1). The yield criterion suggested by Hill $[2,3]$ for a rigid plastic circular plate in terms of the generalized stresses is

$f\left(M_{i j}\right)=C_{1} M_{r}^{2}-C_{2} M_{r} M_{\theta}+C_{3} M_{\theta}^{2}+C_{4} M_{r \theta}^{2}+C_{5} M_{r} M_{r \theta}+C_{6} M_{\theta} M_{r \theta}-h^{4}=0$

in which

$C_{1}=(G+H) \cos ^{4} \theta-\frac{1}{2} H \sin ^{2} 2 \theta+(F+H) \sin ^{4} \theta+\frac{1}{2} N \sin ^{2} 2 \theta$,

$C_{2}=-\frac{1}{2}(G+H) \sin ^{2} 2 \theta+2 H\left(\sin ^{4} \theta+\cos ^{4} \theta\right)-\frac{1}{2}(F+H) \sin ^{2} 2 \theta+N \sin ^{2} 2 \theta$,

$C_{3}=(G+H) \sin ^{4} \theta-\frac{1}{2} H \sin ^{2} 2 \theta+(F+H) \cos ^{4} \theta+\frac{1}{2} N \sin ^{2} 2 \theta$,

$C_{4}=(G+H) \sin ^{2} 2 \theta+2 H \sin ^{2} 2 \theta+(F+H) \sin ^{2} 2 \theta+2 N \cos ^{2} 2 \theta$,

$C_{5}=-2(G+H) \sin 2 \theta \cos ^{2} \theta+H \sin 4 \theta+2(F+H) \sin 2 \theta \sin ^{2} \theta+N \sin 4 \theta$, $C_{\theta}=-2(G+H) \sin 2 \theta \sin ^{2} \theta+H \sin 4 \theta+2(F+H) \sin 2 \theta \cos ^{2} \theta-N \sin 4 \theta$, $k^{2}=h^{4}$.

$$
\begin{aligned}
& 2 F=\frac{1}{Y^{2}}+\frac{1}{Z^{2}}-\frac{1}{X^{2}}, \\
& 2 G=\frac{1}{Z^{2}}+\frac{1}{X^{2}}-\frac{1}{Y^{2}}, \\
& 2 H=\frac{1}{X^{2}}+\frac{1}{Y^{2}}-\frac{1}{Z^{2}}, \\
& 2 N=\frac{1}{T^{2}} .
\end{aligned}
$$

In these equations, $2 h$ is the plate thickness, $X, Y, Z$ are the tensile yield stresses in the principal directions of orthotropy and $T$ is the yield stress in shear with respect to these principal axes.

Lower bound. Assume a stress field

$$
\begin{aligned}
& M_{r}=\frac{1}{6} p m\left(a^{2}-r^{2}\right), \\
& M_{\theta}=\frac{1}{6} p m a^{2}, \\
& M_{r \theta}=0
\end{aligned}
$$

satisfying the stress boundary condition and the equilibrium equation

$$
\frac{d}{d r}\left(r M_{r}\right)-M_{\theta}=-\frac{1}{2} p m r^{2} .
$$

The functional $F^{0}$ defined by (20) becomes in this case

$$
F^{0}=m-\int_{0}^{2 \pi} \int_{0}^{r} \mu\left[C_{1} M_{r}^{2}-C_{2} M_{r} M_{\theta}+C_{3} M_{\theta}^{2}-h^{4}+\phi^{2}\right] r d r d \theta
$$

For a family of orthotropic plates such that 


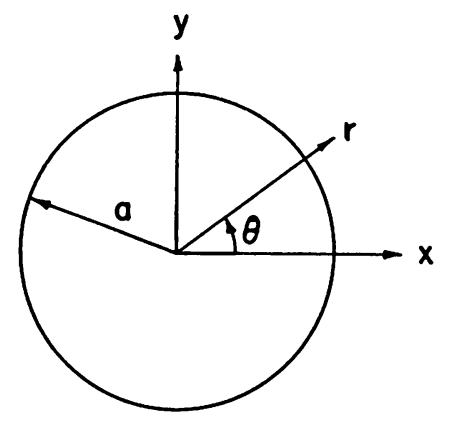

Fig. 1. Circular orthotropic plate.

$$
F=G / \mu_{1}, \quad H=G, \quad N=3 G
$$

in which $\mu_{1}$ is a proportional constant, $C_{1}, C_{2}$ and $C_{3}$ in (32) become

$$
\begin{aligned}
& C_{1}=G\left[2 \cos ^{4} \theta+\sin ^{2} 2 \theta+\left(1+\frac{1}{\mu_{1}}\right) \sin ^{4} \theta\right], \\
& C_{2}=G\left[2\left(\sin ^{4} \theta+\cos ^{4} \theta\right)-\frac{1}{2}\left(\frac{1}{\mu_{1}}-3\right) \sin ^{2} 2 \theta\right], \\
& C_{3}=G\left[2 \sin ^{4} \theta+\sin ^{2} 2 \theta+\left(1+\frac{1}{\mu_{1}}\right) \cos ^{4} \theta\right] .
\end{aligned}
$$

Substituting (34) and (38) in (36), assuming that $\mu=\mu_{0}=$ constant and $\phi=\phi_{0}=$ constant and integrating lead to

$$
\begin{aligned}
F^{0}=m-\mu_{0}\left\{\frac{\pi G a^{6}}{4}\left(\frac{p m}{6}\right)^{2}\right. & {\left[\frac{1}{6}\left(13+\frac{3}{\mu_{1}}\right)\right.} \\
& \left.\left.-\frac{1}{4}\left(18-\frac{2}{\mu_{1}}\right)+\frac{1}{2}\left(13+\frac{3}{\mu_{1}}\right)\right]-h^{4} a^{2} \pi+\phi_{0}^{2} a^{2} \pi\right\} .
\end{aligned}
$$

The values of the unknowns are determined by extremizing $F^{0}$ with respect to $m, \mu_{0}$ and $\phi_{0}$, i.e.,

$$
\partial F^{0} / \partial m=0, \quad \partial F^{0} / \partial \mu_{0}=0 \quad \text { and } \quad \partial F^{0} / \partial \phi_{0}=0 .
$$

Solving these equations yields

$$
\begin{gathered}
m=\frac{12(2)^{1 / 2}}{\left[\frac{25}{6}+\frac{5}{2} \frac{1}{\mu_{1}}\right]^{1 / 2}} \frac{M_{0}}{p a^{2}} \\
\mu_{0}=\frac{6(2)^{1 / 2}}{\left[\frac{25}{6}+\frac{5}{2} \frac{1}{\mu_{1}}\right]^{1 / 2}} \frac{M_{0}}{\pi p a^{4} h^{4}}>0 \\
\phi_{0}=0
\end{gathered}
$$

in which

$$
M_{0}=\frac{h^{2}}{(2 G)^{1 / 2}}=X h^{2}
$$


The constant $\alpha^{0}$ defined by (19) takes the form, in this case,

$$
\alpha^{0}=\left[\frac{h^{4}}{\max \left\{C_{1} M_{r}^{2}-C_{2} M_{r} M_{\theta}+C_{3} M_{\theta}^{2}\right\}}\right]^{1 / 2} .
$$

For a given value of $\mu_{1}, m$ is given by (40), $\max \left\{\frac{1}{2} C_{i j k l} M_{i j} M_{k l}\right\}$ is calculated with the aid of (34), (38) and (40), and $m^{0}$ by (18) and (42). The values of $m^{0}$ are plotted in Fig. 2 against arguments of $\mu_{1}$ in terms of $M_{0} / p a^{2}$.

Upper bound. Assume the velocity field

$$
\dot{w}=A_{1}(a-r)
$$

in which $A_{1}$ is an arbitrary constant, satisfying (10), the boundary condition at $r=a$. Substituting (43) in (11) and solving for $A_{1}$ yields

$$
A_{1}=\frac{3}{\pi p a^{3}} .
$$

The condition (4) becomes in this case

$$
\begin{aligned}
-\dot{w}_{, r r} & =\mu\left(2 C_{1} M_{r}-C_{2} M_{\theta}\right), \\
-\frac{\dot{w}_{. r}}{r} & =\mu\left(2 C_{3} M_{\theta}-C_{2} M_{r}\right) .
\end{aligned}
$$

Solving simultaneously for $M_{r}$ and $M_{\theta}$ with the aid of (43) and (44) and assuming

$$
\mu=\frac{A_{2}}{r}
$$

in which $A_{2}$ is a constant lead to

$$
\begin{aligned}
& M_{r}=\frac{C_{2}}{4 C_{1} C_{3}-C_{2}^{2}} \frac{3}{\pi p a^{3} A_{2}}, \\
& M_{\theta}=\frac{2 C_{1}}{4 C_{1} C_{3}-C_{2}^{2}} \frac{3}{\pi p a^{3} A_{2}} .
\end{aligned}
$$

The functional $F^{*}$ defined by (26) takes the form

$$
\begin{array}{rl}
F^{*}=2 h^{4} \int_{0}^{2 \pi} \int_{0}^{a} \mu r & d r d \theta \\
& +\int_{0}^{2 \pi} \int_{0}^{a} \mu\left[C_{1} M_{r}^{2}-C_{2} M_{r} M_{\theta}+C_{3} M_{\theta}^{2}-h^{4}-\phi^{2}\right] r d r d \theta
\end{array}
$$

Substituting (45) and (46) in (47), assuming $\phi=0$ and integrating yield

$$
F^{*}=4 \pi a h^{4} A_{2}+\frac{9 a N}{A_{2}\left(\pi p a^{3}\right)^{2}}-2 \pi a h^{4} A_{2}
$$

in which

$$
N=\int_{0}^{2 \pi} \frac{C_{1}}{4 C_{1} C_{3}-C_{2}^{2}} d \theta .
$$

Extremizing $F^{*}$ with respect to $A_{2}$, i.e.,

$$
\partial F^{*} / \partial A_{2}=0
$$


and solving for $A_{2}$ yield

$$
A_{2}=\frac{3}{\pi p a^{3} h^{2}}\left(\frac{N}{2 \pi}\right)^{1 / 2}
$$

It should be observed that (45) and (50) satisfy constraint condition (2). Condition (25) in this case takes the form

$$
C_{1} M_{r}^{2}-C_{2} M_{r} M_{\theta}+C_{3} M_{\theta}^{2}=\left(\alpha^{*}\right)^{2} h^{4} .
$$

Solving for $\alpha^{*}$ yield

$$
\alpha^{*}=\frac{\left(C_{1} M_{r}^{2}-C_{2} M_{r} M_{\theta}+C_{3} M_{\theta}^{2}\right)^{1 / 2}}{h^{2}} .
$$

The upper bound of the safety factor $m^{*}$ defined by (24) becomes

$$
m^{*}=2 h^{4} \int_{0}^{2 \pi} \int_{0}^{a} \mu \alpha^{*} r d r d \theta .
$$

Substituting (45) and (51) with the aid of (46) and (50) and integrating yield the upper bound of the safety factor,

$$
m^{*}=\frac{6 h^{2}}{\pi p a^{2}} \int_{0}^{2 \pi}\left(\frac{C_{1}}{4 C_{1} C_{3}-C_{2}^{2}}\right)^{1 / 2} d \theta .
$$

The values of $m^{*}$ are plotted in Fig. 2 against arguments of $\mu_{1}$ in terms of $M_{0} / p a^{2}$.

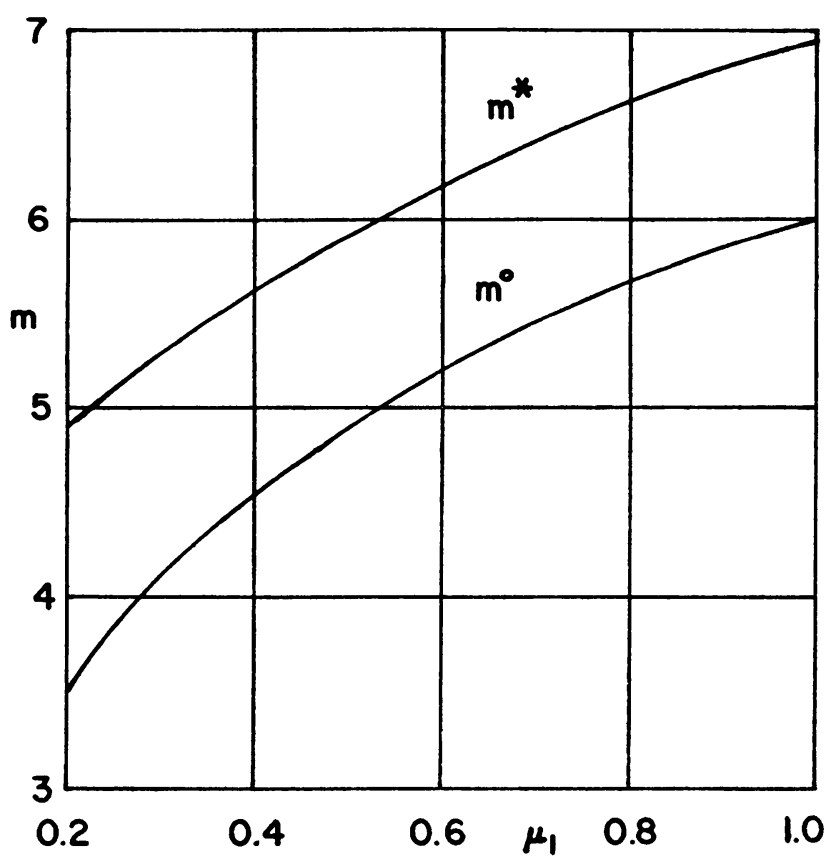

FIG. 2. Values of multipliers plotted against arguments of $\mu_{1}$. 
6. Conclusion. Distinct from the extended theorems of limit analysis presented recently by Mura, Rimawi and Lee [4] in which the yield condition is replaced by a weighted integral mean of the yield function, the present method satisfies all the constraint conditions of the classical theorems. It is of interest to note that the lower and upper bounds obtained by the proposed method are in this instance the same as those obtained by the classical method [5]. In the proposed method, however, the two functionals, $F^{0}$ and $F^{*}$ provide the means by which both the upper and the lower bounds can be obtained by variational procedure. Furthermore it is recalled that, in the lower bound analysis of the classical method, the point at which $\max \left[\frac{1}{2} C_{i j k l} M_{i j} M_{k l}\right]$ occurs has to be guessed a priori in order to satisfy the yield condition $f \leq 0$. Should the wrong assumption be made, the calculation has to be repeated all over again. In the proposed method, on the other hand, this point is located at the end of the procedure after all the constraint conditions have been satisfied, hence no guessing is necessary in this respect. Last, but not the least, it should be mentioned that no functional, such as $F^{0}$, is up to now available for lower bound analysis in the classical method of limit analysis.

\section{REFERENCES}

1. T. Mura and S. L. Lee, Q. Appl. Math., 21, 243 (1963)

2. R. Hill, Proc. Roy. Soc. A, 193, 281 (1948)

3. R. Hill, Mathematical theory of plasticity, Oxford Univ. Press, London, 1950

4. T. Mura, W. H. Rimawi and S. L. Lee, Q. Appl. Math., 23, 171 (1965)

5. T. Mura, J. S. Kao and S. L. Lee, J. Eng. Mech. Div., Proc. ASCE, 90, EM5, 375 (1964) 